IMA Journal of Management Mathematics (2020) 31, 217-231

doi:10.1093/imaman/dpz010

Advance Access publication on 24 July 2019

\title{
Multiple-criteria cash-management policies with particular liquidity terms
}

\author{
Francisco SALAS-Molina $^{\dagger}$ \\ Universitat de València, Av. Tarongers s/n, 46022, Valencia, Spain \\ ${ }^{\dagger}$ Corresponding author. Email: francisco.salas-molina@uv.es \\ David Pla-Santamaria, Ana Garcia-Bernabeu and Fernando Mayor-Vitoria \\ Universitat Politècnica de València, Ferrándiz y Carbonell, 03801, Alcoy, Spain
}

[Received on 23 September 2018; revised on 5 March 2019; accepted on 5 June 2019]

\begin{abstract}
Eliciting policies for cash management systems with multiple assets is by no means straightforward. Both the particular relationship between alternative assets and time delays from control decisions to availability of cash introduce additional difficulties. Here we propose a cash management model to derive shortterm finance policies when considering multiple assets with different expected returns and particular liquidity terms for each alternative asset. In order to deal with the inherent uncertainty about the near future introduced by cash flows, we use forecasts as a key input to the model. We express uncertainty as lack of predictive accuracy and we derive a deterministic equivalent problem that depends on forecasting errors and preferences of cash managers. Since the assessment of the quality of forecasts is recommended, we describe a method to evaluate the impact of predictive accuracy in cash management policies. We illustrate this method through several numerical examples.
\end{abstract}

Keywords: cash flow, forecasting, optimization, multiple criteria decision-making, liquidity terms.

\section{Introduction}

Cash managers have to make daily decisions about the amount of transactions between cash holdings and short-term investment assets. On the one hand, a certain amount of cash must be kept for operational and precautionary purposes. On the other hand, idle cash balances may be invested in a number of alternative assets such as interest-bearing accounts or treasury bills for a profit. Within this context, chances are that a number of assets with different expected returns are available for cash managers. These assets may also present particular liquidity terms meaning that the time period from the selling decision to the availability of cash is not necessarily zero.

Since Miller \& Orr (1966), different models have been proposed to address the cash management problem (CMP) from a stochastic point of view. Gregory (1976), Srinivasan \& Kim (1986) and Costa Moraes et al. (2015) review most of the cash management models proposed in the literature. Typically, cash management models are based on a set of control limits or bounds. Cash balance is then allowed to wander around among some bounds, usually a high bound and a low bound. When any of these bounds is reached, a control action is taken to restore the balance to some target level. For instance, the Miller \& Orr (1966) model is based on three bounds and Eppen \& Fama (1969), Penttinen (1991) and Gormley \& Meade (2007) consider four bounds and use cash flow forecasts as an input to the model.

Multidimensional cash management systems were first considered by Baccarin (2009). The author proposed an impulse control approach restricted to continuous fluctuations of cash balances given by homogeneous diffusion processes. Gormley \& Meade (2007) and Costa Moraes \& Nagano (2014) suggested the use of evolutionary algorithms to derive policies. However, this approach does not 
guarantee the optimality of solutions. The Miller \& Orr (1966) model was extended by Higson et al. (2009) to consider non-stationary cash flow processes following an analytic approach. Another cash management model for banks branches was analytically derived by Cabello (2013) assuming a Poisson process to describe cash demands. This model was later used by Cabello \& Lobillo (2017) to propose an algorithm to optimize branch holdings. On the other hand, the use of particular liquidity terms has been recently introduced in the analysis of alternative policies by Costa Moraes \& Nagano (2014) within the framework of the Miller \& Orr (1966) model. In this paper, we consider liquidity terms, but we follow a different approach by considering cash flow forecasts as a key input to a multicriteria optimization model that can be solved through mathematical programming.

Even though multiple criteria decision-making is a well-established discipline in finance, multiple but possibly conflicting goals in cash management has been recently proposed by Salas-Molina et al. (2018b,a). The authors consider both cost and risk of alternative cash policies under a compromise programming framework. Summarizing, common features of cash management models proposed in the literature include the following: (i) a two-assets setting with a cash account and an investment account; (ii) the assumption of some probability distribution for cash flow processes; (iii) control actions take place instantaneously; and (iv) the minimization of a single goal, namely, cost.

We rely on goal programming (GP) and stochastic goal programming (SGP) to produce cash management policies. GP (Charnes \& Cooper, 1977; Romero, 1991; Aouni et al., 2014) aggregates goals to obtain a solution that minimizes the sum of deviations between achievement and aspiration levels. SGP (Charnes \& Cooper, 1959; Prekopa, 1995; Aouni et al., 2012) is the natural extension of GP to a stochastic context. In order to deal with the inherent uncertainty of cash flows in the near future, we follow the approach of assuming that cash managers can produce forecasts with a given accuracy. Based on previous knowledge about the distribution of past forecasting errors, cash managers set restrictions to policies to ensure that cash balances remain within control limits after actual cash flow realizations. We find applications of SGP in different areas such as portfolio selection, project selection, resource allocation, health care management, transportation and marketing (Aouni et al., 2012). In this paper, we extend the previous range of applications to cash management where uncertainty is limited to future cash flows by means of a model that

1. Generalizes previous approaches by considering particular liquidity terms for each alternative asset.

2. Includes multiple criteria through SGP.

3. Allows one to estimate the impact of predictive accuracy of cash flow forecasts.

In what follows, we provide useful background on the CMP in Section 2. In Section 3, we introduce our multiple criteria model for particular liquidity terms. Next, in Section 4, we describe our approach to dealing with uncertainty in cash management. In Section 5, we illustrate our approach by means of two numerical examples using real data from an industrial company. Finally, in Section 6, we conclude and propose future extensions for this work.

\section{Background: the CMP}

The CMP aims to find a balance between cash holdings and short-term investments. The number of different accounts under consideration, the uncertainty associated to future cash flows for each of the accounts, the transaction and holding costs are key factors that may influence decision-making. In this section, we describe a general optimization problem with applications for both individuals and 
organizations. However, particular cases for the above mentioned factors may lead to optimization problems with a different degree of complexity.

Traditionally, the CMP has been approached from a control perspective. The cash balance of any bank account needs to be monitored to keep the balance among some control bounds. To this end, control actions are taken to increase or decrease the cash balance according to some rules in order to minimize holding and transaction costs. The set of control actions deployed over a planning horizon is called a policy, and it is the solution to the CMP.

Formally, the CMP is usually defined (Gormley \& Meade, 2007; Salas-Molina et al., 2018b) as an optimization problem whose goal is to find the best sequence of transactions summarized in vector $\boldsymbol{x}=\left[x_{1}, \ldots, x_{t}, \ldots, x_{T}\right]$, with $x_{t} \in \mathbb{R}, t \in \mathbb{N}$ and $1 \leqslant t \leqslant T$ for a given planning horizon $T \in \mathbb{N}$. Balances at the end of the day are computed as the sum of the previous cash balance $b_{t-1}$, the actual cash flow $f_{t}$ and the control action $x_{t}$, according to the following state transition law

$$
b_{t}=b_{t-1}+f_{t}+x_{t}
$$

where $b_{t}, f_{t}, x_{t} \in \mathbb{R}$, with stochastic cash flows $f_{t}$ following some probability distribution, either theoretical or empirical.

Within the common two-assets setting, the CMP is characterized by its particular cost structure. Any ordering transaction into a cash account may have a cost, which may include a fixed part $\left(\gamma_{0}^{+}\right)$and a variable part $\left(\gamma_{1}^{+}\right)$. On the other hand, a return transaction from a cash account may also have a cost with a fixed part $\left(\gamma_{0}^{-}\right)$and a variable part $\left(\gamma_{1}^{-}\right)$. Furthermore, at the end of the day, a holding cost $(h)$ per money unit is charged if a positive cash balance occurs, or a penalty cost $(u)$ per money unit is charged if a negative cash balance occurs. According to this cost structure, a general daily cost function is defined as

$$
c\left(x_{t}\right)=\Gamma\left(x_{t}\right)+L\left(b_{t}\right)
$$

where $x_{t}$ is the transaction made at day $t, b_{t}$ is the cash balance at the end of day $t, \Gamma\left(x_{t}\right)$ is a transfer cost function and $L\left(b_{t}\right)$ stands for a holding/shortage cost function. The transfer cost function $\Gamma\left(x_{t}\right)$ is defined as:

$$
\Gamma\left(x_{t}\right)= \begin{cases}\gamma_{0}^{-}-\gamma_{1}^{-} \cdot x_{t} & \text { if } x_{t}<0, \\ 0 & \text { if } x_{t}=0, \\ \gamma_{0}^{+}+\gamma_{1}^{+} \cdot x_{t} & \text { if } x_{t}>0 .\end{cases}
$$

Additionally, the holding/shortage cost function is expressed as

$$
L\left(b_{t}\right)= \begin{cases}-u \cdot b_{t} & \text { if } b_{t}<0 \\ v \cdot b_{t} & \text { if } b_{t}>0\end{cases}
$$

where $u$ is the shortage cost and $v$ is the holding cost per money unit held in cash. Under this cost structure, the ultimate goal of cash managers is typically to find the policy $\boldsymbol{x}$ that minimizes the total cost over the planning horizon $T$

$$
C(\boldsymbol{x})=\sum_{t=1}^{T} c\left(x_{t}\right)=\sum_{t=1}^{T}\left[\Gamma\left(x_{t}\right)+L\left(b_{t}\right)\right]
$$




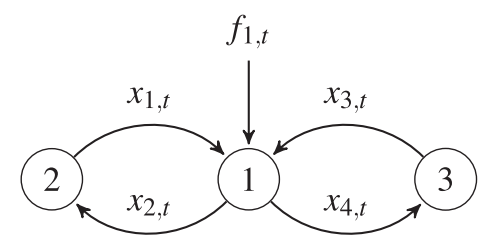

FIG. 1. A cash management system with three bank accounts.

This formulation is restricted to the common assumptions present in the cash management literature. In this paper, we propose a more general formulation to account for multiple assets with particular liquidity terms within a multiple criteria decision-making framework.

\section{Dealing with particular liquidity terms}

In this section, we rely on GP (Charnes \& Cooper, 1977; Ballestero \& Romero, 1998; Ballestero, 2001; Abdelaziz et al., 2007; Aouni et al., 2014) to reformulate the CMP for multiple assets with different returns and particular liquidity terms. More precisely, we use SGP to minimize both the cost and the risk of alternative cash management policies as recently proposed by Salas-Molina (2017) and SalasMolina et al. (2018b). Since cash flows are usually affected by some uncertainty, our model is designed for a stochastic context.

Cash managers usually have alternative investment options for idle balances. Then, cash management systems with multiple assets are very common in practice. In addition, alternative assets may have both different expected returns and particular liquidity terms. We here define liquidity terms as the necessary number of time steps from the selling decision to the availability of cash. As an example, consider the cash management system described in Costa Moraes \& Nagano (2014) depicted in Fig. 1. Circles stand for bank accounts and directed arrows between circles standing for transactions. This cash management system consists of a main bank account 1 , receiving external net cash flows $f_{t}$, and two alternative investment assets, namely, assets 2 and 3. Each investment asset $i$ presents a particular expected return $r_{i}$, and each transaction $j$ presents a liquidity term $d_{j}$, usually expressed in days. For instance, asset 2 may be an interest bearing account returning an $r_{2}=2 \%$ interest rate per year with full liquidity for increasing and decreasing decisions, i.e. $d_{1}, d_{2}=0$, and asset 3 may be a treasury bill returning an $r_{3}=4 \%$ interest rate per year with selling liquidity term $d_{3}=2$ days due to market restrictions, but no delay for buying decisions, i.e. $d_{4}=0$.

Cash managers must find a balance between holding and transaction costs. They also have to include in the analysis both the expected returns and the liquidity terms of alternative assets since some decisions imply a delay in the availability of funds. At each time step $t$, four possible transactions $x_{j, t}$ are available to control cash balances. As a result, the state transition law in equation (1) can be generalized to any cash management system with $m$ bank accounts and $n$ possible transactions with, at most, $D$ different liquidity terms by means of the following system of linear equations:

$$
\boldsymbol{b}_{t}=\boldsymbol{b}_{t-1}+\boldsymbol{f}_{t}+\sum_{k=0}^{D} A_{k} \boldsymbol{x}_{t-k}
$$

where $\boldsymbol{b}_{t-1}$ and $\boldsymbol{b}_{t}$ are $m \times 1$ vectors with previous and current balances for each account, respectively, and $f_{t}$ is an $m \times 1$ vector with external net cash flows for each account. The most right-hand side term of equation (6) summarizes all possible transactions with possible delays between zero and a maximum 
value $D$ that depends on the particular liquidity terms of alternative assets. Furthermore, $A_{k}$ is an $m \times n$ matrix with element $a_{i j}$ set to the following: 1 if transaction $j$ adds cash to account $i$ at time $t$ due to a decision made $k$ time steps ago; -1 if transaction $j$ removes cash from account $i$ at time $t$ due to a decision made $k$ time steps ago; 0 otherwise. Finally, $\boldsymbol{x}_{t-k}$ is an $n \times 1$ vector with the set of transactions (control actions) decided at time $t-k$.

In the example in Fig. 1 , if $d_{1}, d_{2}, d_{4}=0$ and $d_{3}=2$ days, then $D=2$ and

$$
A_{0}=\left[\begin{array}{rrrr}
1 & -1 & 0 & -1 \\
-1 & 1 & 0 & 0 \\
0 & 0 & 0 & 1
\end{array}\right] ; A_{1}=0 ; A_{2}=\left[\begin{array}{rrrr}
0 & 0 & 1 & 0 \\
0 & 0 & 0 & 0 \\
0 & 0 & -1 & 0
\end{array}\right]
$$

Simple algebra leads to the following system of linear equations equivalent to equation (6):

$$
\begin{aligned}
& b_{1, t}=b_{1, t-1}+f_{1, t}+x_{1, t}-x_{2, t}+x_{3, t-2}-x_{4, t} \\
& b_{2, t}=b_{2, t-1}-x_{1, t}+x_{2, t} \\
& b_{3, t}=b_{3, t-1}-x_{3, t-2}+x_{4, t} .
\end{aligned}
$$

Within this framework, when a selling decision $x_{3, t}$ is made, the current cash balance in accounts 1 and 3 is not updated until the amount is finally transferred 2 days later. When the particular law of motion for a given cash management system is established, we are in a position to linearize the cost functions described in Section 2 in order to formulate the CMP as a linear program. Following SalasMolina (2017), we rewrite the transaction cost function in equation (3) as follows:

$$
\Gamma\left(\boldsymbol{x}_{t}\right)=\boldsymbol{\gamma}_{0}^{\prime} \cdot z_{t}+\boldsymbol{\gamma}_{1}^{\prime} \cdot \boldsymbol{x}_{t}
$$

subject to

$$
\boldsymbol{x}_{t} \leqslant M \cdot z_{t}
$$

where $z_{t}$ is an $n \times 1$ binary vector with element $z_{j}$ set to one if the $j$-th element of $\boldsymbol{x}_{t}$ is not null, and 0 otherwise; $\boldsymbol{\gamma}_{0}$ is an $n \times 1$ vector of fixed transaction costs for each transaction; $\boldsymbol{\gamma}_{1}$ is an $n \times 1$ vector of variable transaction costs and $M$ is a very large number. Note that liquidity terms do not affect transaction costs since these are charged when the decision is made.

For optimization purposes, we need additional exclusivity constraints that avoid bidirectional transactions at the same time step. For example, it does not make sense that $x_{1, t}$ and $x_{2, t}$ in Fig. 1 occur at the same time step. We can exclude this bidirectional transaction by setting an exclusivity constraint such as $z_{1, t}+z_{2, t} \leqslant 1$. When dealing with different liquidity terms, we have also to consider the corresponding delays between decisions. For example, avoiding that $x_{3, t-2}$ and $x_{4, t}$ occur simultaneously. To facilitate the understanding of the links established between assets, we propose to represent cash management systems as directed graphs $G=(V, E)$ with a set of accounts $V$ and a set of transactions $E$ such that each transaction $x_{i, t}=(p, q)$ is defined by a tail account $p \in V$, which is the origin of funds, and a head account $q \in V$, which is the destination of funds. Then for each pair of accounts $p, q \in V$, if there is a pair of transactions $x_{i, t}, x_{j, t} \in E$ such that $x_{i, t}=(p, q)$ and $x_{j, t}=(q, p)$, we set exclusivity constraints

$$
z_{i, t}+z_{j, t} \leqslant 1 \quad \forall t \in 1,2, \ldots T
$$


Generalizing to the case of particular liquidity terms between $k=0$ and a maximum of $D$ time steps, we extend the representation of transactions to consider liquidity terms as a tuple $x_{i, t}=\left(p, q, d_{i}\right)$, where $d$ is the required time steps between a decision and the availability of funds in the destination account. As a result, for each pair of accounts $p, q \in V$, if there is a pair of transactions $x_{i, t}, x_{j, t} \in E$ such that $x_{i, t}=\left(p, q, d_{i}\right)$ and $x_{j, t}=\left(q, p, d_{j}\right)$, then we set the following exclusivity constraints

$$
z_{i, t-d_{i}}+z_{j, t-d_{j}} \leqslant 1 \quad \forall t \in \max \left(d_{i}, d_{j}\right)+1, \ldots, T
$$

Note that we restrict these exclusivity constraints to the case of positive time index $t$. For ease of notation, we summarize all the exclusivity constraints in the following set of linear equations:

$$
H \cdot z \leqslant \mathbf{1}
$$

where $H$ is a $T \cdot n \times T \cdot n$ matrix of zero-one coefficients, $z$ is a $T \cdot n \times 1$ vector of binary variables obtained through vertical concatenation of daily vectors $z_{t}$ and $\mathbf{1}$ is a $T \cdot n \times 1$ vector of ones.

On the other hand, the holding cost function in equation (4) can be expressed as

$$
L\left(b_{t}\right)=v^{\prime} \cdot b_{t} \cdot y_{t}+u^{\prime} \cdot b_{t} \cdot\left(1-y_{t}\right)
$$

where $\boldsymbol{V}$ is an $m \times 1$ column vector with the $i$-th element set either to the holding cost $v_{i}$ or the expected return $r_{i}$ multiplied by -1 since it is a profit instead of a cost for account $i$; $\boldsymbol{u}$ is an $m \times 1$ column vector with the $i$-th element set to the penalty cost for account $i ; \boldsymbol{y}_{t}$ is an $m \times 1$ binary vector with element $y_{i}$ set to one if the $i$-th element of $\boldsymbol{b}_{t}$ is non-negative, and 0 otherwise; and $\mathbf{1}$ is an $m \times 1$ column vector of ones. Note that equation (16) is still non-linear since we are multiplying two decision variables $\boldsymbol{b}_{t}$ and $\boldsymbol{y}_{t}$. This equation can also be linearized by means of additional auxiliary variables as described in Bemporad \& Morari (1999). Nevertheless, since cash managers usually discard policies with negative balances due to high penalty costs for negative cash balances, in what follows, we assume $\boldsymbol{y}_{t}=\mathbf{1}$ and $L\left(\boldsymbol{b}_{t}\right)=\boldsymbol{v}^{\prime} \cdot \boldsymbol{b}_{t}$. This assumption is equivalent to the constraint $\boldsymbol{b}_{t} \in \mathbb{R}_{\geqslant 0}^{m}$ and implies that policies with negative cash balances are infeasible.

Within the typical single objective framework, given an initial cash balance $\boldsymbol{b}_{0}$, and a cash flow $\boldsymbol{f}_{t}$ for $1 \leqslant t \leqslant T$, the solution to the CMP for multiple assets and particular liquidity terms is a $T \cdot n \times 1$ policy vector $\boldsymbol{x}=\left[\boldsymbol{x}_{1}, \boldsymbol{x}_{2}, \ldots, \boldsymbol{x}_{T}\right]^{\prime}$, obtained through vertical concatenation of daily vectors $\boldsymbol{x}_{t}$ that minimizes cost goal $g_{1}(\boldsymbol{x})$ as the sum of transaction and holding costs up to time step $T$ :

$$
\min g_{1}(\boldsymbol{x})=\min \sum_{t=1}^{T} c\left(\boldsymbol{x}_{t}\right)=\min \sum_{t=1}^{T}\left(\boldsymbol{\gamma}_{0}^{\prime} \cdot \boldsymbol{z}_{t}+\boldsymbol{\gamma}_{1}^{\prime} \cdot \boldsymbol{x}_{t}+\boldsymbol{v}^{\prime} \cdot \boldsymbol{b}_{t}\right)
$$

subject to

$$
\begin{gathered}
\boldsymbol{x} \in S^{T \times n} \\
z \in\{0,1\}^{T \times n} \\
\boldsymbol{b}_{t} \in \mathbb{R}_{\geqslant 0}^{m},
\end{gathered}
$$

where $S^{T \times n}$ is the set of all feasible solutions given by the constraints of the problem for the whole planning horizon $T$. 
On the other hand, cash managers may be interested not only in the cost but also in the risk associated with alternative polices as suggested by Salas-Molina et al. (2018b). In this work, the authors proposed the use of the standard deviation and the upper semideviation of daily costs as a measure of risk. Herrera-Caceres \& Ibeas (2016) used a predefined cash balance signal as a way to control balances. Salas-Molina (2017) proposed the use of cash balance deviations from a given reference as a measure of risk. Within the typical GP framework, we here follow Salas-Molina (2017) to include an additional risk goal $g_{2}\left(\boldsymbol{x}_{t}\right)$ expressed as the sum of balances of the system

$$
g_{2}\left(\boldsymbol{x}_{t}\right)=\boldsymbol{\beta}^{\prime} \cdot \boldsymbol{b}_{t},
$$

where $\boldsymbol{\beta}$ is and $m \times 1$ vector of zero-one coefficients reflecting the interest of cash managers to measure risk by controlling balances of either all accounts or a subset of accounts. For example, if we want to control balances only for account 1 in Fig. 1 , we set $\boldsymbol{\beta}=[1,0,0]^{\prime}$. The rationale behind the choice of cash balance deviations as a measure of risk is its linearity and the possibility to be straightforwardly integrated within a GP approach. Alternative risk measures used in finance and cash management such as value-at-risk and standard deviation are non-linear, hence compromising tractability due to the need of non-linear optimization algorithms. A further motivation of the use of cash balance deviations as a measure of risk is the concept of stability. We argue that stable policies, namely, those with balances that deviate less from a given reference, better capture the notion of risk in cash management.

Cash managers aim to minimize the sum of positive and negative deviations from a given balance reference $b_{r e f} \in \mathbb{R}$ subject to the typical GP constraint

$$
g_{2}(\boldsymbol{x})-\delta_{2 t}^{+}+\delta_{2 t}^{-}=b_{r e f}
$$

This risk goal can be easily accommodated in the classical GP approach. As a result, we propose the following model to solve the CMP for multiple assets with particular liquidity terms within a cost-risk minimization framework

$$
\min \frac{w_{1}}{C_{\max }} \sum_{t=1}^{T} \delta_{1 t}^{+}+\frac{w_{2}}{R_{\max }} \sum_{t=1}^{T}\left(\delta_{2 t}^{+}+\delta_{2 t}^{-}\right)
$$

subject to

$$
\begin{gathered}
\boldsymbol{b}_{t}=\boldsymbol{b}_{t-1}+\boldsymbol{f}_{t}+\sum_{k=0}^{D} A_{k} \boldsymbol{x}_{t-k} \\
\boldsymbol{x}_{t} \leqslant M \cdot \boldsymbol{z}_{t} \\
H \cdot \boldsymbol{z} \leqslant \mathbf{1} \\
\boldsymbol{\gamma}_{0}^{\prime} \cdot \boldsymbol{z}_{t}+\boldsymbol{\gamma}_{1}^{\prime} \cdot \boldsymbol{x}_{t}+\boldsymbol{v}^{\prime} \cdot \boldsymbol{b}_{t}-\delta_{1 t}^{+} \leqslant 0 \\
\boldsymbol{\beta}^{\prime} \cdot \boldsymbol{b}_{t}-\delta_{2 t}^{+}+\delta_{2 t}^{-}=b_{r e f} \\
\boldsymbol{x}_{t} \in \mathbb{R}_{\geqslant 0}^{n} \\
z \in\{0,1\}^{T \times n} \\
\boldsymbol{b}_{t} \in \mathbb{R}_{\geqslant 0}^{m} \\
\delta_{1 t}^{+}, \delta_{2 t}^{+}, \delta_{2 t}^{-} \geqslant 0
\end{gathered}
$$




$$
\begin{gathered}
1 \leqslant t \leqslant T \\
t, n, T, D \in \mathbb{N},
\end{gathered}
$$

where $\delta_{1 t}^{+}$are auxiliary deviation variables for daily cost measured by the sum of transaction and holding costs, and $\delta_{2 t}^{-}, \delta_{2 t}^{+}$are auxiliary deviation variables for daily risk measured by the sum of total balance deviations from reference $b_{r e f}$. Note that the target in constraint (27) is zero since we want to minimize daily costs. Then negative deviation variables are also zero, i.e. $\delta_{1 t}^{-}=0$. Similarly, bluethe goal in equation (28) ensures that aggregated balances of the system and daily deviations result in target $b_{r e f}$. Weights $w_{1}$ and $w_{2}$ reflect preferences for cost and risk of cash managers and $C_{\max }$ and $R_{\max }$ are used to avoid numerical bias for one of the goals (Ballestero \& Romero, 1998). In our context, it is likely that cash balances in goal 2 are much higher than daily costs in goal 1. To avoid biased results towards the goal that can achieve higher values, namely, cash balances, objective function (23) is normalized by measuring goal achievement as a percentage of $C_{\max }$ and $R_{\max }$. These values can also be considered as budget limits for both cost and risk by setting additional constraints of the type

$$
\begin{gathered}
\sum_{t=1}^{T} \delta_{1 t}^{+} \leqslant C_{\text {max }} \\
\sum_{t=1}^{T} \delta_{2 t}^{-}+\delta_{2 t}^{+} \leqslant R_{\text {max }}
\end{gathered}
$$

discarding solutions exceeding budget limits $C_{\max }$ and $R_{\text {max }}$.

\section{Dealing with uncertainty through SGP}

Note that the GP model described in Section 3 implies that cash flow $\boldsymbol{f}_{t}$ is known with certainty for the whole planning horizon $T$. When dealing with stochastic (but possibly predictable) cash flows, a variation of this model can be considered to account for the inherent uncertainty of cash flows through SGP (Prekopa, 1995; Abdelaziz et al., 2007; Aouni et al., 2012). The cash management model described in Section 3 can be extended to a stochastic context by means of the following program:

$$
\min g(\boldsymbol{x})
$$

subject to

$$
\begin{aligned}
& \sum_{j=1}^{n} a_{i j} x_{j} \geqslant \tilde{h}_{i} \\
& i=1,2, \ldots, s,
\end{aligned}
$$

where uncertainty is limited to random variable $\tilde{h}_{i}$ for each one of the $s$ constraints of the problem. This structure fits well in the CMP where random variable $\tilde{h}_{i}$ maps each one of the predicted cash flows $\tilde{f}_{i}$ that are affected by some prediction error. Let us assume that cash managers can produce cash flow forecasts subject to some prediction error. By considering the probability distribution of the uncertainty produced by prediction errors, the cash management model can be reformulated as a Chance Constrained 
Program (CCP) as proposed by Charnes \& Cooper (1959). A CCP can be converted into its deterministic counterpart as follows (Prekopa, 1995):

$$
\min \mathrm{E}[g(\boldsymbol{x})]
$$

subject to

$$
\mathrm{P}\left(\sum_{j=1}^{n} a_{i j} x_{j} \geqslant \tilde{f}_{i}\right) \geqslant \xi_{i},
$$

where $\xi_{i}$ are probability parameters for each of the constraints that are specified by cash managers. In our context, equation (41) is the stochastic equivalent of equation (24):

$$
\mathrm{P}\left(\tilde{\boldsymbol{b}}_{t} \geqslant \tilde{\boldsymbol{b}}_{t-1}+\sum_{k=0}^{D} A_{k} \boldsymbol{x}_{t-k}+\tilde{\boldsymbol{f}}_{t}\right) \geqslant \xi
$$

and it should be read as follows: the probability that cash balances are greater or equal to initial balances, control actions $\boldsymbol{x}_{t-k}$ and random cash flows $\tilde{\boldsymbol{f}}_{t}$ is at least $\xi$, which we assume to be constant without loss of generality. Based on previous knowledge about past forecasting errors, cash managers set parameter $\xi$ to build a deterministic equivalent problem ensuring that cash balances after control actions and cash flow realizations remain within some control limits with probability $\xi$. The higher the value of $\xi$, the more conservative the cash management policy since higher cash balances would be required to ensure that random cash flows do not produce undesired deviations.

Note that balances $\tilde{\boldsymbol{b}}_{t}$ and cash flows $\tilde{\boldsymbol{f}}_{t}$ are now random variables. In this paper, we assume that cash managers can produce cash flow forecasts with a given prediction error. Then random variable $\tilde{f}_{t}$ can be expressed in terms of a time series forecast $\hat{\boldsymbol{f}}_{t}$, and an $m$-dimensional error term $\boldsymbol{\varepsilon}_{t}$

$$
\tilde{f}_{t}=\hat{f}_{t}+\varepsilon_{t}
$$

Since actual cash balances are also affected by the same forecasting errors according to $\boldsymbol{b}_{t}=\hat{\boldsymbol{b}}_{t}+\boldsymbol{\varepsilon}_{t}$, random balances $\tilde{\boldsymbol{b}}_{t}$ and random cash flows $\tilde{\boldsymbol{f}}_{t}$ are replaced with predicted balances $\hat{\boldsymbol{b}}_{\boldsymbol{t}}$ and cash flow forecasts $\hat{f}_{t}$ to build the deterministic equivalent CMP through the following constraint:

$$
\mathrm{P}\left(\hat{\boldsymbol{b}}_{t} \geqslant \hat{\boldsymbol{b}}_{t-1}+\sum_{k=0}^{D} A_{k} \boldsymbol{x}_{t-k}+\hat{\boldsymbol{f}}_{t}\right) \geqslant \xi
$$

The higher the uncertainty about the near future, the higher the forecasting error. With perfect information about the near future, the probability that predicted cash balances $\hat{\boldsymbol{b}}_{t}$ are greater or equal to a given minimum balance threshold determined by initial balances $\hat{\boldsymbol{b}}_{t-1}$, control actions $\boldsymbol{x}_{t-k}$ and predicted cash flows $\hat{\boldsymbol{f}}_{t}$ is one. When some prediction error $\boldsymbol{\varepsilon}_{t}$ is assumed, this balance threshold should be higher to ensure that equation evaluated in (44) holds with probability $\xi$. In other words, cash managers aim to ensure that actual balances after cash flow error realization are above balances computed with perfect 
information. This strategy is equivalent to add minimum cash balance constraints of the type

$$
\hat{\boldsymbol{b}}_{t} \geqslant \boldsymbol{b}_{\min }
$$

where $\boldsymbol{b}_{\text {min }}$ is an $m$-dimensional vector of minimum cash balances for each account that ultimately depends on parameter $\xi$. For instance, starting from an initial balance of zero monetary units and assuming daily Gaussian forecasting errors expressed as $\mathrm{N}(0,1)$, with zero mean and unit standard deviation, if we set parameter $\xi=99 \%$, the cash balance constraint of an account should be greater than $b_{\text {min }}=3$ to avoid that an unexpected negative swift brings the balance below the starting balance. If we set parameter $\xi=95 \%$, the cash balance constraint should be greater than $b_{\min }=2$ according to the normal probability distribution.

However, note that constraints of the type of equation (45) are hard constraints, i.e. those that have to be satisfied whatever the realization of the underlying uncertainty. This fact may lead to unfeasible policies even in the case that expected balances are below the established minimums but very close to them. GP allows us to overcome this problem by transforming hard constraints such as equation (45) into an additional risk goal $g_{2}(\boldsymbol{x})$ from equation (22) measuring cash balance variability from a given reference.

An additional advantage of our model in comparison to the approach followed by Gormley \& Meade (2007) and Costa Moraes \& Nagano (2014), based on evolutionary algorithms to solve the CMP, is the optimality of solutions. It is known that heuristics such as evolutionary algorithms do not guarantee optimal solutions. On the contrary, the deterministic equivalent of our SGP model can be solved by means of commercial mathematical programming solvers such as Gurobi or CPLEX ensuring optimality. The presence of binary variables may lead to computationally expensive problems in the case of large instances. However, in the context of cash management with short-term planning horizons, the size of optimization problems is likely to be computationally tractable. Indeed, Salas-Molina et al. (2018c) report run times below tenths of a second for integer linear programs for CMPs with planning horizons between 5 and 20 days. In addition, they report run times several orders of magnitude faster than an evolutionary algorithm. Summarizing, our SGP approach combined with common mathematical programming solvers represents an advantage in terms of tractability of problems and optimality of solutions.

\section{The impact of cash flow predictive accuracy}

In this section, we provide several numerical examples to illustrate the utility of our multiple criteria cash management model for particular liquidity terms. We consider two likely scenarios: one in which cash flows are known with certainty and another one in which cash flows can be predicted with some accuracy. In order to allow comparisons with recent related work such as Costa Moraes \& Nagano (2014), we experiment on the cash management system described in Fig. 1 in which temporary idle cash balances in account 1 can be invested in two alternative assets 2 and 3. Expected returns and transaction costs are selected from those proposed in Costa Moraes \& Nagano (2014) summarized in the cost structure in Table 1.

Note that the expected return for each alternative asset is indeed a negative cost within the context of a minimization problem such as the one encoded from equations 2334. Finally, we consider variable transaction costs for buying decisions lower than variable costs for selling decisions in order to guarantee that the unitary cost of transferring money to any of the investments is lower than the profit obtained (Constantinides \& Richard, 1978). 
TABLE 1 Cost structure data for the examples

\begin{tabular}{lccccc}
\hline Transaction & $\gamma_{0}(\backslash$ euro $)$ & $\gamma_{1}(\%)$ & $d$ (days) & Account & $r(\%)$ \\
\hline 1 & 50 & 0.01 & 0 & 1 & 0 \\
2 & 50 & 0.001 & 0 & 2 & 0.003 \\
3 & 50 & 0.01 & 2 & 3 & 0.005 \\
4 & 50 & 0.001 & 0 & & \\
\hline
\end{tabular}

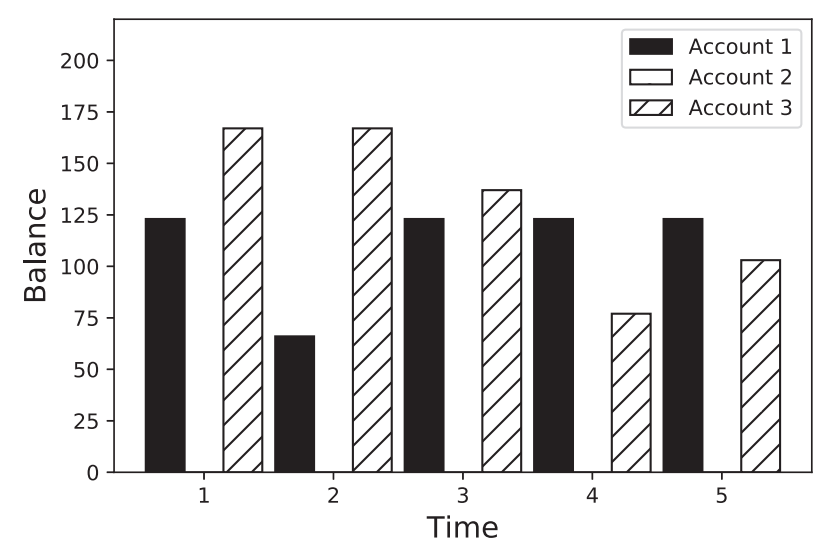

FIG. 2. Balances derived for the optimal policy with perfect information.

\subsection{Planning with perfect information about future cash flows}

In a cash management context, the assumption of perfectly known cash flows is by no means as restrictive as it may seem at first glance. On the contrary, cash flows for short-term planning horizons, e.g. the next five working days, can be known with certainty in many cases. The existence of the socalled major cash flows (Stone \& Miller, 1987) such as loan payments, taxes, dividends or the monthly payroll makes near-to-perfect predictions a suitable option.

As a first illustrative example, assume that the cash flow for the next five days in account 1 from Fig. 1 is $f_{1}=[10,-57,27,-60,26]^{\prime}$, all figures in thousands of Euros. We set $C_{\text {max }}$ to the opportunity cost of cash holdings in account 1 , which we estimate as the returns that could be obtained by allocating all expected balances in account 3 that yields the maximum return within the system. In this example, we are interested in measuring risk as the cash balance variation in account 1 since it is the main cash account in the system. Then we set $\boldsymbol{\beta}=[1,0,0]^{\prime}$. Assume that from the analysis of past observations of the underlying cash flow process, we set cash balance reference $b_{\text {ref }}=123$ as an acceptable cash balance for operational purposes. From that reference, we set $R_{\text {max }}=b_{r e f} \cdot T$ proportional to both cash balance reference and planning horizon $T$. Note that this procedure to set $b_{r e f}$ and $R_{\max }$ is a practical rule and that many other rules would also be possible.

Consider a hypothetical cash manager without preference for cost and risk $\left(w_{1}, w_{2}=0.5\right)$. The solution for the deterministic model of this numerical example under the cost structure defined in Table 1 for cash flow $\boldsymbol{f}_{1}$ and an stable initial condition $\boldsymbol{b}_{0}=[200,20,60]^{\prime}$ is shown in Fig. 2 and the set of transactions in Fig. 3.

From Fig. 2, one can observe that idle cash balances in account 1 are invested in account 3 instead of account 2 due to the higher return until is again required at time 4 because of the high negative cash 


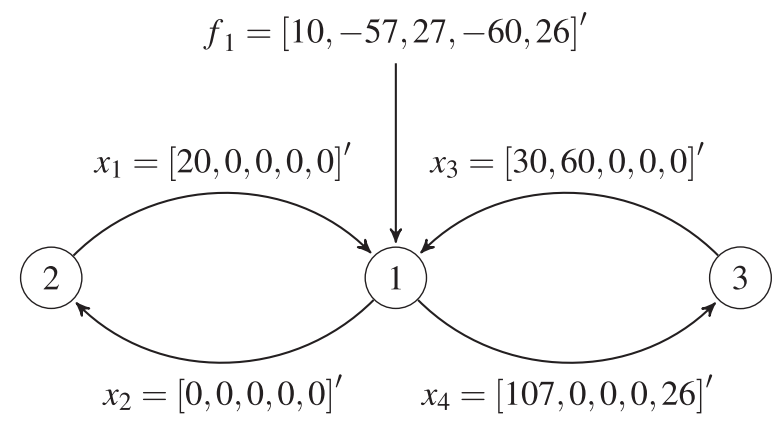

FIG. 3. Example of the optimal policy for a given cash flow.

flow at that time. From Fig. 3, further insights on the issue of liquidity terms for alternative investment accounts addressed in this paper can be obtained. Indeed, selling decisions $x_{3,1}=30$ and $x_{3,2}=60$ are made at time 1 and 2 in order to face the high negative cash flow at time 2 and 4 . Since the liquidity term for investment account 3 is 2 days, the decision is made in advance in order to guarantee the feasibility of the policy for the whole planning horizon. Even though the decision is made at times 1 and 2, cash balance in both accounts 1 and 3 is updated 2 days later as shown in Fig. 2.

\subsection{Planning using cash flow forecasts with a given predictive accuracy}

In the previous example, we assumed that perfect short-term cash flow predictions can be procured. If this is not the case, our model formulation can also be applied by replacing cash flow and balances by their respective forecasts. Several works have demonstrated the utility of cash flow forecasting in cash management (Stone, 1972; Miller \& Stone, 1985; Gormley \& Meade, 2007; Salas-Molina et al., 2017). These results must encourage cash managers to drive their efforts to achieve better forecasts.

In order to deal with the inherent uncertainty of future cash flows, we rely on SGP to solve the CMP. Within a context affected by forecasting errors, deploying a policy obtained using our model implies that actual cash balances are random variables of the form $\tilde{\boldsymbol{b}}_{t}=\hat{\boldsymbol{b}}_{t}+\boldsymbol{\varepsilon}_{t}$, which are affected by some prediction error. The deterministic equivalent of the SGP described in Sections 3 and 4 requires replacing actual balances and cash flows with their respective forecasts $\hat{\boldsymbol{b}}_{t}$ and $\hat{\boldsymbol{f}}_{t}$, and setting a balance reference $b_{r e f}$

$$
\min \frac{w_{1}}{C_{\max }} \sum_{t=1}^{T} \delta_{1 t}^{+}+\frac{w_{2}}{R_{\max }} \sum_{t=1}^{T}\left(\delta_{2 t}^{+}+\delta_{2 t}^{-}\right)
$$

subject to

$$
\begin{gathered}
\hat{\boldsymbol{b}}_{t}=\hat{\boldsymbol{b}}_{t-1}+\hat{\boldsymbol{f}}_{t}+\sum_{k=0}^{D} A_{k} \boldsymbol{x}_{t-k} \\
\boldsymbol{x}_{t} \leqslant M \cdot z_{t} \\
H \cdot \boldsymbol{z} \leqslant \mathbf{1} \\
\boldsymbol{\gamma}_{0}^{\prime} \cdot z_{t}+\boldsymbol{\gamma}_{1}^{\prime} \cdot \boldsymbol{x}_{t}+\boldsymbol{v}^{\prime} \cdot \hat{\boldsymbol{b}}_{t}-\delta_{1 t}^{+} \leqslant 0 \\
\boldsymbol{\beta}^{\prime} \cdot \hat{\boldsymbol{b}}_{t}-\delta_{2 t}^{+}+\delta_{2 t}^{-}=b_{r e f} .
\end{gathered}
$$


TABLE 2 Impact of forecasting error results

\begin{tabular}{lcc}
\hline Error distribution & $\mathcal{N}(0,10)$ & $\mathcal{N}(0,80)$ \\
\hline Number of replicates & 1000 & 1000 \\
Planning horizon $(T)$ & 5 & 5 \\
Difference in average objective function $(\%)$ & 4 & 36 \\
Difference in standard deviation & 0.02 & 0.05 \\
Difference in average cost $(\%)$ & 8 & 59 \\
Difference in average risk $(\%)$ & 0 & 13 \\
\hline
\end{tabular}

The solution of the previous cash management model is a policy based on forecasts affected by some prediction error that ultimately results in a cash balance deviation of magnitude equal to the error for each time step. Given a probability distribution for error process $\boldsymbol{\varepsilon}_{t}$ (for instance, Gaussian), we can evaluate its impact on actual costs by randomly drawing a number of realizations of this error distribution that we later use to compute both actual balances and model performance. The following experimental procedure evaluates the impact of predictive accuracy in the performance of policies by measuring cost and risk but with actual cash balances:

1. Randomly sample $T$ cash flows from the data set to obtain $f_{t}$.

2. Obtain policies and balances by solving the deterministic equivalent problem in (46)-(51).

3. Sample $T$ errors from $\mathcal{N}\left(0, \sigma_{e}\right)$ for account affected by forecasting uncertainty to obtain $\boldsymbol{\varepsilon}_{t}$ for $1 \leqslant t \leqslant T$.

4. Compute actual cash balances as $\tilde{\boldsymbol{b}}_{t}=\hat{\boldsymbol{b}}_{t}+\boldsymbol{\varepsilon}_{t}$ and actual performance through objective function (46).

For the sole purpose of the following example, let us consider that policies for a planning horizon of 5 days cannot be modified. In practice, cash managers would compute a new policy on a daily basis to achieve the desired feedback as recommended in Bemporad \& Morari (1999). However, the assumption of a fixed policy allows us to evaluate the impact of forecasting errors on actual performance. To this end, we experiment on a real cash flow data set from an industrial company with 1000 observations with mean 8.3 and standard deviation 123, figures in thousands of Euros. Assume that we can produce forecasts for account 1 with errors described by a Gaussian distribution $\mathcal{N}\left(0, \sigma_{e}\right)$. A value of $\sigma_{e}=10$ can be considered a good prediction since actual observations present a remarkably higher standard deviation. On the contrary, a value of $\sigma_{e}=80$ can be considered a bad prediction for similar reasons.

Consider again the cash management system depicted in Fig. 1 and the cost structure in Table 1. In both cases, we consider planning horizon $T=5$ days and the same procedure described in Section 5.1 to select parameters $C_{\max }, R_{\max }$ and $b_{\text {ref }}$. For comparative purposes, we start both experiments with the same initial balance for account 1 of three times the standard deviation of the cumulative cash flow for planning horizon $T$. For accounts 2 and 3, initial cash balances are set to zero. After 1000 replicates of the previous procedure for $\sigma_{e}=10$ and 80 produced the results summarized in Table 2. After computing actual costs, we obtained differences in average costs and standard deviation of costs with respect to a benchmark using perfect predictions, i.e. with no errors.

As expected, the lack of knowledge about the future reduces the possibility of making good decisions in advance. The results show that lower forecasting errors lead to lower variations with respect to the perfect information case described in Section 5.1. Specific results for both cost and risk show that an 
increase in predictive accuracy (expressed as a reduction in the standard deviation of errors from 80 to 10) leads to a reduction in the cost of policies (from 59 to 8) but removes all risk (from 13 to 0 ) with respect to the case with perfect information. Beyond the expected results of better performance for better predictive accuracy, cash managers can rely on the procedure described in this section to evaluate the impact of an increase in predictive accuracy in both cost and risk of cash management policies. This analysis guides cash managers in answering critical questions about the use of forecasting techniques in cash management. An example of these questions is if efforts dedicated to improve predictive accuracy by using new forecasting techniques match increased performance of policies. Summarizing, the results presented in this section must encourage cash managers to produce better forecasts to be used as a key input to our model for multiple assets and particular liquidity terms.

\section{Concluding remarks}

Motivated by the idea of providing better policies within the context of cash management systems with multiple assets and particular liquidity terms, this paper presents a multiple criteria GP model to solve the CMP subject to these common features. In addition, we provide cash managers with the possibility to consider cash flow forecasts to reduce the uncertainty about the near future. As a result, we include both time delays derived from liquidity terms and cash flow forecasts as key inputs to derive satisficing solutions within a GP framework.

To consider not only the cost of alternative policies but also the risk, we propose a multiple criteria GP model with both cost and risk as goals to minimize. We use cash balance deviations as a measure of risk and a GP approach by transforming hard constraints in soft ones by means of SGP. As a result, we provide a more flexible method to overcome the drawback of considering hard minimum balance constraints that have to be satisfied whatever the realization of the underlying uncertainty.

The critical step in the deployment of our model is the procurement of reliable short-term cash flow forecasts. In order to analyse the impact of forecasting errors in the cost of policies, we report an experimental procedure that can be used to estimate actual performance within a stochastic context. As expected, the higher the predictive accuracy of forecasts, the higher the benefits that derive from our model. This fact must encourage researchers to produce better time-series forecasting techniques since accuracy is highly rewarded.

Further research may consider alternative approaches to consider both totally random cash flows and multiple assets with particular liquidity terms along the lines discussed in Section 3. In practice, this could help integrate the management of both cash flows known with certainty and random cash flows. Finally, exploring formal methods to specify the parameters of the model such as goal weights according to the preferences of cash managers rather than relying on subjective judgments is also an interesting future line of work.

\section{REFERENCES}

Abdelaziz, F. B., Aouni, B. \& El Fayedh, R. (2007) Multi-objective stochastic programming for portfolio selection. Eur. J. Op. Res. , 177, 1811-1823.

Aouni, B., Ben Abdelaziz, F. \& La Torre, D. (2012). The stochastic goal programming model: theory and applications. J. Multi-Criteria Decis. Anal. , 19, 185-200.

Aouni, B., Colapinto, C. \& La Torre, D. (2014). Financial portfolio management through the goal programming model: current state-of-the-art. Eur. J. Op. Res., 234, 536-545.

BACCARIN, S. (2009). Optimal impulse control for a multidimensional cash management system with generalized cost functions. Eur. J. Oper. Res., 196, 198-206. 
BALLESTERo, E. (2001). Stochastic goal programming: a mean-variance approach. Eur. J. Oper. Res., 131, 476-481.

Ballestero, E. \& Romero, C. (1998). Multiple criteria decision making and its applications to economic problems. New York: Springer Science \& Business Media

Bemporad, A. \& Morari, M. (1999). Control of systems integrating logic, dynamics, and constraints. Automatica J. IFAC, 35, 407-427.

Cabello, J. G. (2013). Cash efficiency for bank branches. SpringerPlus, $2,334$.

Cabello, J. G. \& Lobillo, F. (2017). Sound branch cash management for less: a low-cost forecasting algorithm under uncertain demand. Omega, 70, 118-134.

Charnes, A. \& Cooper, W. W. (1959). Chance-constrained programming. Manag. Sci, 6, 73-79.

Charnes, A. \& CoOPER, W. W. (1977). Goal programming and multiple objective optimizations. Eur. J. Oper. Res., $1,39-54$.

Constantinides, G. M. \& Richard, S. F. (1978). Existence of optimal simple policies for discounted-cost inventory and cash management in continuous time. Oper. Res., 26, 620-636.

Da Costa Moraes, M. B. \& Nagano, M. S. (2014). Evolutionary models in cash management policies with multiple assets. Econ. Model., 39, 1-7.

da Costa Moraes, M. B., Nagano, M. S. \& Sobreiro, V. A. (2015) Stochastic cash flow management models: a literature review since the 1980s. Decision Models Eng Manag. New York: Springer International Publishing, pp. 11-28.

EpPEn, G. D. \& FAMA, E. F. (1969) Cash balance and simple dynamic portfolio problems with proportional costs. Internat. Econom. Rev., 10, 119-133.

Gormley, F. M. \& Meade, N. (2007). The utility of cash flow forecasts in the management of corporate cash balances. Eur. J. Op. Res., 182, 923-935.

Gregory, G. (1976) Cash flow models: a review. Omega, 4, 643-656.

HerRera-CaCERES, C. A. \& IBEAs, A. (2016) Model predictive control of cash balance in a cash concentration and disbursements system. J. Franklin Institute, 353, 4885-4923.

Higson, A., Yoshikatsu, S. \& Tippett, M. (2009) Organization size and the optimal investment in cash. IMA. J. Manag. Math., 21, 27-38.

Miller, M. H. \& OrR, D. (1966) A model of the demand for money by frms. Q. J. Econom., 80, 413-435.

Miller, T. W. \& Stone, B. K. (1985) Daily cash forecasting and seasonal resolution: alternative models and techniques for using the distribution approach. J. Financial Q. Anal., 20, 335-351.

Penttinen, M. J. (1991) Myopic and stationary solutions for stochastic cash balance problems. Eur. J. Op. Res., 52, 155-166.

Prekopa, A. (1995) Stochastic programming. Dordrecht: Kluwer Academic Publishers.

Romero, C. (1991) Handbook of critical issues in goal programming. Oxford: Pergamon Press.

Salas-Molina, F. (2017). Risk-sensitive control of cash management systems. Oper. Res., in press.

Salas-Molina, F., Martin, F. J., Rodriguez-Aguilar, J. A., Serra, J. \& Arcos, J. L. (2017). Empowering cash managers to achieve cost savings by improving predictive accuracy. Int. J. Forecast, 33, 403-415.

Salas-Molina, F., Pla-Santamaria, D. \& Rodriguez-Aguilar, J. A. (2018a). A multi-objective approach to the cash management problem. Annals Op Res, 267, 515-529.

Salas-Molina, F., Pla-Santamaria, D. \& Rodriguez-Aguilar, J. A. (2018b). Empowering cash managers through compromise programming. Financial Decision Aid Using Multiple Criteria. Cham: Springer, pp. 149-173.

Salas-Molina, F., Rodriguez-Aguilar, J. A. \& Pla-Santamaria, D. (2018c). Boundless multiobjective models for cash management. Eng. Econ., 63, 363-381.

SRinivasan, V. \& Kim, Y. H. (1986). Deterministic cash ow management: state of the art and research directions. Omega, 14, 145-166.

Stone, B. K. (1972). The use of forecasts and smoothing in control-limit models for cash management. Financial Manag., 1, 72-84.

Stone, B. K. \& Miller, T. W. (1987). Daily cash forecasting with multiplicative models of cash flow patterns. Financial Manag., 16, 45-54. 